\title{
Influence of the crown-to-implant length ratio on the clinical performance of implants supporting single crown restorations: a cross-sectional retrospective 5-year investigation
}

\author{
Schneider, D ; Witt, Lukas Andreas ; Hämmerle, C H F
}

\begin{abstract}
Purpose: The aim of this study was to investigate the influence of the crown-to-implant length ratio (c/i ratio) on the implant survival, changes of the marginal bone level (MBL) and the occurrence of biological and technical complications. Material and methods: This cross-sectional retrospective study included all patients with implants in the posterior segments supporting single crown restorations with a minimum follow-up of 5 years. All patients were questioned and examined clinically and radiographically. The technical and biological c/i ratio and the MBL were measured on digitized periapical radiographs. The following outcome parameters in relation to the $\mathrm{c} / \mathrm{i}$ ratio and the co-factors were statistically analyzed: implant survival rate, MBL, occurrence of technical and biological complications. For statistical analysis, regression, correlation and survival analyses were applied $(\mathrm{P}<0.05)$. Results: Seventy patients (mean age of 50.7 years [range 19.8-76.6 years]) with a total of 100 implants $(24$ Straumann type, 76 Brånemark type) were included in this study. The mean follow-up period was 6.2 years (range 4.73-11.7 years). Six implants failed during the follow-up period, yielding a cumulative survival rate of $95.8 \%$ at 5 years in function. The mean technical c/i ratio was $1.04( \pm 0.26$, range $0.59-2.01)$. The mean biological c/i ratio was 1.48 ( \pm 0.42 , range $0.82-3.24)$. No statistically significant influence of the technical and biological c/i ratio was found on the implant survival, MBL and occurrence of technical and biological complications. When adjusted for the biological c/i ratio, smoking was the only co-factor significantly associated with implant failure and biological complications. Conclusion: In the present study, the $\mathrm{c} / \mathrm{i}$ ratio did not influence the clinical performance of implants supporting single crown restorations in the posterior segments of the jaw within the range tested. To cite this article: Schneider D, Witt L, Hämmerle CHF. Influence of the crown-to-implant length ratio on the clinical performance of implants supporting single crown restorations: a cross-sectional retrospective 5-year investigation. Clin. Oral Impl. Res. 23, 2012; 169-174. doi: 10.1111/j.1600-0501.2011.02230.x.
\end{abstract}

DOI: https://doi.org/10.1111/j.1600-0501.2011.02230.x

Posted at the Zurich Open Repository and Archive, University of Zurich

ZORA URL: https://doi.org/10.5167/uzh-58273

Journal Article

Accepted Version

Originally published at:

Schneider, D; Witt, Lukas Andreas; Hämmerle, C H F (2012). Influence of the crown-to-implant length ratio on the clinical performance of implants supporting single crown restorations: a cross-sectional retrospective 5-year investigation. Clinical Oral Implants Research, 23(2):169-174.

DOI: https://doi.org/10.1111/j.1600-0501.2011.02230.x 


\section{Influence of the crown-to-implant length ratio on clinical performance of implants supporting single crown restorations - a cross-sectional retrospective five-year investigation}

David Schneider ${ }^{\text {a }}$, Lukas Witt ${ }^{\text {}}$, Christoph H. F. Hämmerle ${ }^{c}$,

a) Senior lecturer, Center for Dental and Oral Medicine and Cranio-Maxillofacial Surgery, Clinic of Fixed and Removable Prosthodontics and Dental Material Science, University of Zurich, Switzerland

b) Undergraduate student, Center for Dental and Oral Medicine and Cranio-Maxillofacial Surgery, University of Zurich, Switzerland

c) Professor and Chairman, Center for Dental and Oral Medicine and Cranio-Maxillofacial Surgery, Clinic of Fixed and Removable Prosthodontics and Dental Material Science, University of Zurich, Switzerland

Address for correspondence:

Dr. med., Dr. med. dent. David Schneider

Clinic of Fixed and Removable Prosthodontics and Dental Material Science

University of Zurich

Plattenstrasse 11

8032 Zurich

Switzerland

E-mail: david.schneider@zzm.uzh.ch

Phone: +41446343251 


\section{Abstract}

Purpose: The aim of this study was to investigate the influence of the crown-to-implant length ratio (c/i ratio) on implant survival, changes of the marginal bone level and the occurrence of biological and technical complications. Material and methods: This crosssectional retrospective study included all patients with implants in the posterior segments supporting single crown restorations with a minimum follow-up of five years. All patients were questioned and clinically and radiographically examined. The technical and biological c/i-ratio and the marginal bone level (MBL) were measured on digitized periapical radiographs. The following outcome parameters in relation to the c/i ratio and the co-factors were statistically analyzed: implant survival rate, marginal bone level, occurrence of technical and biological complications. For statistical analysis, regression, correlation and survival analyses were applied $(p<0.05)$. Results: Seventy patients (mean age of 50.7 years (range 19.8 - 76.6 years) with a total of 100 implants (24 Straumann type, 76 Brånemark type) were included in this study. The mean follow-up period was 6.2 years (range 4.73 - 11.7 years). Six implants failed during the follow-up period, yielding a cumulative survival rate of $95.8 \%$ at 5 years in function. The mean technical c/i ratio was $1.04( \pm 0.26$, range $0.59-2.01)$. The mean biological c/i ratio was $1.48( \pm 0.42$, range 0.82 - 3.24). No statistically significant influence of the technical and biological c/i ratio was found on implant survival, marginal bone level and occurrence of technical and biological complications. When adjusted for biological c/i ratio smoking was the only co-factor significantly associated with implant failure and biological complications. Conclusion: In the present study, the c/i ratio did not influence the clinical performance of implants supporting single crown restorations in the posterior segments of the jaw within the range tested. 


\section{Introduction}

In patients with reduced periodontal attachment prosthetic reconstructions are often characterized by a long clinical crown and small amount of intraalveolar root anchorage. It had historically been assumed that, based on the lever principle, the resulting forces on the remaining attachment are unfavorable regarding the prognosis of the abutment tooth. Almost a century ago a dogmatic guideline for the prosthetic rehabilitation of partially edentulous patients was posted claiming that "the total periodontal membrane area of the abutment teeth should equal or exceed that of the teeth to be replaced" (Ante 1926). However, different studies showed that teeth with a reduced but healthy periodontium exhibiting a seemingly unfavorable crown-to-root ratio (c/r ratio) can successfully function as abutment teeth (Nyman \& Ericsson 1982; Laurell et al. 1991; Yi et al. 1995).

Masticatory function can be established and maintained independently of the periodontal history of a healthy abutment tooth. Survival rates of reconstructions on healthy teeth with and without a history of periodontitis have been shown to be similar (Lulic et al. 2007). Accordingly, the crown-to-root ratio does not influence the clinical performance of tooth-supported reconstructions under healthy conditions.

A similar clinical situation regarding the crown-to-root length ratio is often encountered in edentulous areas restored with implant supported reconstructions. Due to vertical loss of the alveolar bone after tooth extraction (Schropp et al. 2003; Araujo \& Lindhe 2005), the supracrestal part of the implant borne reconstruction is often long in relation to the clinical crowns of the remaining dentition and to the supporting implant.

Despite the findings in the above-mentioned studies with natural dentitions, clinicians tend to insert the longest implants possible, presuming a higher success rate with increasing crown-to-implant length ratio (c/i ratio). 
The results of studies investigating the influence of the $\mathrm{c} / \mathrm{i}$ ratio on the outcome of implant treatment are rather heterogeneous. Some investigators reported a positive correlation between increased c/i ratio and higher risk for per-implant marginal bone loss (Rangert et al. 1997; Wang 2002), while others failed to show such a correlation (Tawil et al. 2006; Blanes 2009) and even observed an inverse relationship between c/i ratio and marginal bone loss (Blanes et al. 2007).

Studies performed on this topic usually analyze the influence of the c/i ratio on the marginal bone level and the implant survival rate, but only one study also evaluated the occurrence of technical complications (Tawil et al. 2006). Unfortunately, no effort was made to detect a possible correlation between the reported technical complications and the c/i ratio. Moreover, no data are available on how different prosthetic designs (e.g. single crown, splinted crowns, cantilevers), the implant position within the dental arch and other co-factors (e.g. implant type, implant dimension, bruxism, smoking, history of periodontitis) influence the relationship between c/i ratio and marginal bone loss, implant survival rate, as well as occurrence of technical and biological complications. As a consequence, more information is necessary to understand the influence of the $\mathrm{c} / \mathrm{i}$ ratio on the outcome of different implant treatment modalities.

Therefore, the objectives of the present study were:

1) to test whether or not a higher $\mathrm{c} / \mathrm{i}$ ratio is associated with lower implant survival, higher marginal bone loss, and higher occurrence of biological and technical complications, and

2) to test the effect of site- and patient-related co-factors on the outcome. 


\section{Material and Methods}

\section{Study population}

This cross-sectional retrospective study included all patients treated at the Clinic of Fixed and Removable Prosthodontics and Dental Material Science at the University of Zurich, Switzerland, between 1994 and 2004, who fulfilled the following criteria:

- one or more implant(s) in the posterior maxilla or mandible

- these implants supporting single crown restorations

- at least five years between insertion of the reconstruction and the follow-up examination

No restrictions were made regarding implant type or implant dimensions, implants embedded in native or regenerated bone, the mode of retention (cement- or screwretained), the presence of bruxism, smoking or history of periodontitis. All patients were invited by phone or letter to attend the follow-up examination.

\section{Follow-up examination}

All follow-up examinations were performed by one examiner. Patients were questioned using a standardized protocol to obtain information about patient-related co-factors (smoking habits, bruxism and history of periodontitis). In addition, patients were questioned regarding the occurrence of technical and/or biological complications or reinterventions during the loading period. All patient records were screened to evaluate patient- and site-related co-factors such as implant type, implant diameter, retention mode and peri-implant GBR procedures as well as complications during and after implant treatment.

Restorations and implants were clinically examined for signs of technical and biological 
complications.

Technical complications included

- excessive occlusal wear of reconstructive materials

- fracture or chipping of the veneering material

- fracture of the implant

- $\quad$ fracture of the crown framework

- loosening or fracture of the abutment or occlusal screw

- loss of retention of the crown

Biological complications were defined as signs of peri-implant mucosal inflammation (swelling, redness, bleeding on probing, suppuration) and an increased probing depth (4mm or more) in connection with structured parts of the implant, including implant threads, or surface accessible by probing.

The position of the implant restoration defined as whether or not being in terminal position was noted. Moreover, the nature of the opposing dentition was recorded and categorized as natural dentition, tooth-supported fixed prostheses, implant-supported fixed prostheses or removable denture.

\section{Radiographic analysis}

For the evaluation of the crown-to-implant ratio (c/i) and the marginal bone level (MBL), periapical radiographs were taken with the long-cone paralleling technique with the central beam aiming at the alveolar crest (Updegrave 1968). The images were digitized for measuring. For all measurements, the distance of three implant threads was used as the basis for the calibration and determination of the exact magnification and distortion of the images (Rodoni et al. 2005; Benic et al. 2009) (Fig. 1). All radiographic measurements 
were performed by two examiners. In case of disagreement, the values were discussed until a consensus was found.

The length of the implant was measured from the apex to the top of the implant shoulder (Figs. 1 and 2). The length of the crown was measured from the top of the implant shoulder to the most occlusal point. The marginal bone level (MBL) was measured at the mesial and distal aspect of the implant using x10-15 magnification (Buser et al. 1991; Weber et al. 1992). It was defined as the distance between the top of the implant shoulder and the first visible bone-to-implant contact (Fig. 1). For statistical analysis, the mesial and distal values of the MBL were averaged to one value and the marginal bone loss was calculated as the difference between initial mean MBL and the mean MBL at the follow-up examination.

Depending on the outcome measure, two different values of $c / i$ ratio were determined and adapted according to a previous study (Blanes al. 2007) (Fig. 2):

1. The technical $\mathrm{c} / \mathrm{i}$ ratio was determined for the occurrence of technical complications. The top of the implant shoulder was used as transition between the crown and the implant.

2. For the marginal bone loss, implant survival and the occurrence of biological complications, the biological c/i ratio was determined. The reference used for the calculation was the initial peri-implant marginal bone level.

\section{Statistical analysis}

The following primary and secondary predictors were evaluated regarding their impact on the outcome parameters:

Primary predictor: c/i ratio. 
Secondary predictors: Influence of implant type, implant diameter, retention mode, terminal position, opposing dentition, GBR procedure, smoking habits, bruxism and history of periodontitis on the outcome.

Outcome parameters

- Implant survival

- Marginal bone loss

- Occurrence of technical and biological complications (binary variable)

The patient was the statistical unit for the evaluation of the patient-related predictors (bruxism, smoking, history of periodontitis) on the outcome parameters. The implant was the statistical unit for the evaluation of the crown-to-implant ratio on the outcome parameters.

\section{Descriptive statistics}

Mean values, standard deviations and ranges were computed and visualized by histograms for all continuous variables and relative frequencies for all discrete variables.

\section{Comparative statistics}

Due to a narrow distribution of the technical and biological c/i ratios (Figs. 3 and 4), no subgrouping was performed (e.g. c/i ratio $<1$ and $>1$ ).

Cox regression analysis was run to investigate the association between biological c/i ratio and survival of the implants until implant loss. Hazard ratios (HR) were computed together with the corresponding 95\% confidence interval (Cl).

Non-parametric Spearman correlation was applied in order to disclose associations between two continuous variables. Fisher exact test was used in order to disclose 
associations between two discrete variables.

Univariate and multiple logistic regression analyses were conducted to evaluate the influence of the primary and secondary predictors on the occurrence of technical and biological complications. Odds ratios were computed together with the corresponding $95 \% \mathrm{Cl}$.

Results of the statistical analyses were considered significant with $p$-values $<0.05$. All statistical analyses were performed using a statistical software program (PASW Statistics 18.0 for Mac). 


\section{Results}

Seventy patients with a total of 100 dental implants were analyzed in this study. The mean follow-up period amounted to 6.2 years (range 4.73 to 11.7 years).

The study population consisted of 27 men (37\%) and 43 women (63\%) with a mean age of 50.7 years (range 19.8 to 76.6 years). Forty-nine implants (49\%) were located in the premolar region, 51 (51\%) had been placed in the molar area. Thirty implants $(30 \%)$ were positioned in a terminal arch position. Seventy-six implants (76\%) were two-piece implants (Brånemark, Nobel Biocare ${ }^{\mathrm{TM}}$, Gothenburg, Sweden), 24 (24\%) were one-piece implants (Straumann Standard or Standard Plus, Institut Straumann AG, Basel, Switzerland). The mean implant length was $11.5 \mathrm{~mm}$ (median $11.5 \mathrm{~mm}$, min. $7 \mathrm{~mm}$, max. $15 \mathrm{~mm})$. Most of the implants (66\%) had a "regular" diameter (3.75 to $4.1 \mathrm{~mm})$, the others (34\%) were "wide" diameter implants (4.8 $\mathrm{mm}$ to $5.0 \mathrm{~mm}$ ). Forty-six implants $(46 \%)$ were placed in connection with a peri-implant guided bone regeneration (GBR) procedures treating buccal dehiscence type defects, $12(12 \%)$ in connection with a simultaneous sinus floor elevation procedure (Summers technique or lateral antrostomy). Thirty-eight (38\%) implants were placed without augmentative procedures.

After a mean healing time of 12 months (median 9 months, range 10 days to 36 months), the implants were either restored with screw-retained (26\%) or cement-retained $(74 \%)$ single porcelain-fused-to-metal crowns.

The mean technical crown-to-implant ratio was $1.04( \pm 0.26$, median 1.02 , range 0.59 to 2.01; Fig. 3a and b). The mean biological crown-to-implant ratio was $1.48( \pm 0.42$, median 1.43 , range 0.82 to 3.24 ; Fig. $4 a$ and b).

In the opposing jaw, a natural dentition or tooth-supported fixed prostheses were present 
in 54 patients (76\% of the implants), implant-supported fixed restorations in 9 patients (15\% of the implants), and removable dentures in 4 patients (6\% of the implants). In 3 patients ( $3 \%$ of the implants) the situation of the opposing dentition could not be evaluated.

Patient interviews revealed 31 patients (44.3\%) to be smokers and $17(24.3 \%)$ to be bruxers. Fourteen patients (20\%) had a history of periodontitis. In these patients, implants had only been placed after successful treatment of the periodontal disease. These patients had been included in a structured health care follow-up program.

\section{Implant survival}

During the follow-up period, $6(6 \%)$ implants were lost due to peri-implantitis in four patients after $1.1,4.6,5.0,5.7$ and 9.2 years in function, yielding a cumulative survival rate of $95.8 \%$ at 5-years. One lost implant measured $8.5 \mathrm{~mm}$ in length, three $10 \mathrm{~mm}$, one $11.5 \mathrm{~mm}$ and one $13 \mathrm{~mm}$. Three of these patients loosing five implants were smokers. None of the patients had a history of periodontitis. Four of the implants were placed without any bone augmentation procedures, one in connection with a sinus floor elevation and one with GBR due to a dehiscence type defect. The healing time before loading was 0.76 to 2.1 years.

Although, Cox regression analysis revealed a higher biological c/i ratio to negatively be associated with implant failure $(B=-0.15, H R=0.87,95 \% C l(H R)[0.11,7.00])$, this association was not statistically significant.

When adjusted for biological c/i ratio, smoking was significantly associated with implant failure $(B=2.755, H R=15.7,95 \% \mathrm{Cl}(H R)[1.7,139.5], p=0.013)$. 
When adjusted for the biological c/i ratio, none of the following parameters were significantly associated with implant failure: implant diameter, GBR procedures, retention mode, terminal position, bruxism, history of periodontitis, the type of manufacturer.

\section{Marginal bone loss}

The mean marginal bone loss was $-0.008 \mathrm{~mm}$ (SD $0.74 \mathrm{~mm}$, median $-0.009 \mathrm{~mm}$, range 2.13 to $+2.62 \mathrm{~mm}$; Fig. 5).

Spearman correlation analysis revealed no relationship between biological c/i ratio and marginal bone loss $(\mathrm{rho}=0.181, \mathrm{p}=0.081)$.

When adjusted for the biological c/i ratio, none of the following parameters were significantly associated with marginal bone loss: manufacturer, diameter, GBR procedures, retention mode, terminal position, nature of opposing dentition, smoking, bruxism, history of periodontitis.

\section{Technical complications}

Technical complications were observed in 13 of the patients (18.6 \%) and 13 implant reconstructions. Two implants in two patients (2.9\%) experienced two types of technical complications and one implant in one patient (1.4\%) three types of technical complications (Table 1).

Although, logistic regression analysis showed a lower technical c/i ratio to result in more technical complications $(B=-2.61, \mathrm{OR}=0.073,95 \% \mathrm{Cl}(\mathrm{OR})[0.005,1.147], \mathrm{p}=0.063)$, this relationship was not statistically significant.

When adjusted for the technical c/i ratio, none of the following parameters were 
significantly associated with an increased occurrence of technical complications:

Manufacturer, implant diameter, GBR procedures, retention mode, terminal position, type of antagonist, bruxism, history of periodontitis.

\section{Biological complications}

Biological complications occurred at 11 implants (11\%) in 11 patients (15.7\%).

Logistic regression revealed no association between the biological c/i ratio and the occurrence of biological complications $(B=-0.23, \mathrm{OR}=0.795,95 \% \mathrm{Cl}(\mathrm{OR})[0.17,3.712]$, $\mathrm{p}=0.77)$.

When adjusted for biological c/i ratio, smoking was significantly associated with biological complications $(\mathrm{B}=2.668$, $\mathrm{OR}=14.404,95 \% \mathrm{Cl}(\mathrm{OR})[2.861,72.512], \mathrm{p}=0.001)$.

When adjusted for the biological c/i ratio, none of the following parameters were significantly associated with an increased occurrence of biological complications: Manufacturer, implant diameter, GBR procedures, retention mode, terminal position, type of antagonist, bruxism, history of periodontitis. 


\section{Discussion}

The results of the present study showed neither the technical nor the biological c/i ratio to have an effect on the clinical performance of the implants. Only smoking in combination with increased biological $\mathrm{c} / \mathrm{i}$ lead to more implant failures and enhanced the chance for the occurrence of biological complications. No associations were found with any of the other factors investigated.

In the present study no association between the c/i ratio and the implant survival rate was found and the cumulative survival rate reached $95.8 \%$ at 5 years of function. In a systematic review a similar survival rate of $96.8 \%$ was reported at 5 years of loading for implant supported single crowns (Jung et al. 2008). Hence, the implant survival rate in the present study compares well with the bulk of published data. In addition, recent studies investigating survival rates of implant-supported prostheses with increased c/i ratios also showed similar implant survival ranging from $94.1 \%$ to $98.2 \%$ after at least 2 years of function (Blanes 2009; Schulte et al. 2007). Hence, recent studies investigating the association between the implant failure rate and the $\mathrm{c} / \mathrm{i}$ ratio was found no association and have reported survival rates similar to the general literature.

Regarding the amount of marginal bone loss no correlation was found with a higher $\mathrm{c} / \mathrm{i}$ ratio, which is in agreement with the findings in previous studies (Tawil et al. 2006; Blanes 2009). The observed mean marginal bone loss is within the range of previous investigations reporting a mean loss of marginal bone around two-piece implants supporting single tooth restorations of $-0.11 \mathrm{~mm}$ after 5 years in function (Wennstrom et al. 2005) and $-0.15 \mathrm{~mm}$ around one-piece implants (Bornstein et al. 2005). Most patients showed no notable loss of marginal bone. Only four implants in four different patients experienced a loss of bone amounting to 1.5 to $2 \mathrm{~mm}$. These sites were associated with 
clinical and radiographic signs of peri-implantitis. None of these four implants, however, was lost during the observation period.

The occurrence of technical and biological complications in the present study is in agreement with a previous systematic review on complication rates of implant supported single crowns (Jung et al. 2008). No association between biological c/i ratio and biological complications was observed, while the incidence of technical complications tended to decrease with a higher technical c/i ratio. This latter observation, however, is difficult to explain and should be interpreted with caution since no other investigation is available for comparison in the literature.

Among the investigated patient related factors possibly influencing the outcome only smoking in connection with increased biological c/i ratio was found to be associated with more implant failures and more biological complications. The negative effect of smoking on implant survival and peri-implant mucosal health has well been documented in numerous investigations (Strietzel et al. 2007; Gruica et al. 2004; Ortorp \& Jemt 2004). Based on the lever principle it is a common conception that short implants in combination with long suprastructures are more prone to biological (e.g. marginal bone loss, implant disintegration) and technical (e.g. fractures of implant or prosthetic components) complications. Compared to shorter implants studies using finite element analysis showed implants of greater length to alter the stress distribution within the implant and the surrounding bone (Pierrisnard et al. 2003; Koca et al. 2005; Georgiopoulos et al. 2007). The clinical relevance of these findings is yet to be determined.

Many studies on clinical performance of implants report implant survival rates, bone level alterations, occurrence of technical and biological complications with respect to different implant and prosthetic designs. In contrast, surprisingly few articles consider the c/i 
ratio and its influence on the outcome of implant treatment (Blanes et al. 2007; Rokni et al. 2005; Tawil et al. 2006; Schulte et al. 2007). These articles describe the relationship between c/i ratio and implant survival or marginal bone loss. However, very heterogeneous samples were included in these studies regarding implant location and prosthetic design of the suprastructure rendering sound conclusions difficult.

The present study specifically assessed the influence of the $c / i$ ratio on the clinical performance of implants supporting single crown restorations in the posterior segments of the jaw. Only implants supported single crown restorations were included to avoid bias caused by stress distribution of splinted implants and only restorations in the posterior segments were chosen under the assumption of higher occlusal forces and therefore potentially higher risk for complications. In addition, patient- and site-related co-factors were taken into consideration.

The conclusions of this investigation are limited by its retrospective study design, its a narrow distribution of the c/i ratio of included implants, and its relative small number of complications limiting the possibility for statistical analyses. Therefore, further studies are necessary to more clearly define the effect of the c/i ratio on the clinical performance of implant supported restorations with different indications, prosthetic designs and clinical situations. 


\section{Conclusion}

Within the limitations of the present study and the range tested, it can be concluded that the $\mathrm{c} / \mathrm{i}$ ratio did not influence the clinical performance of implants. Therefore, implant restorations with increased c/i ratio may be successfully be used in the posterior region. In contrast to these results, smoking in combination with increased c/i ratio lead to more implant failures and biological complications. Consequently, the use of implant restorations with high c/i ratios may be recommended for single tooth reconstructions.

However, further studies, preferably including higher c/l ratios, are indicated. 


\section{Acknowledgments}

The authors would like to acknowledge Dr. Malgorzata Roos for her support in the statistical analysis of the data. 


\section{Figures and tables}

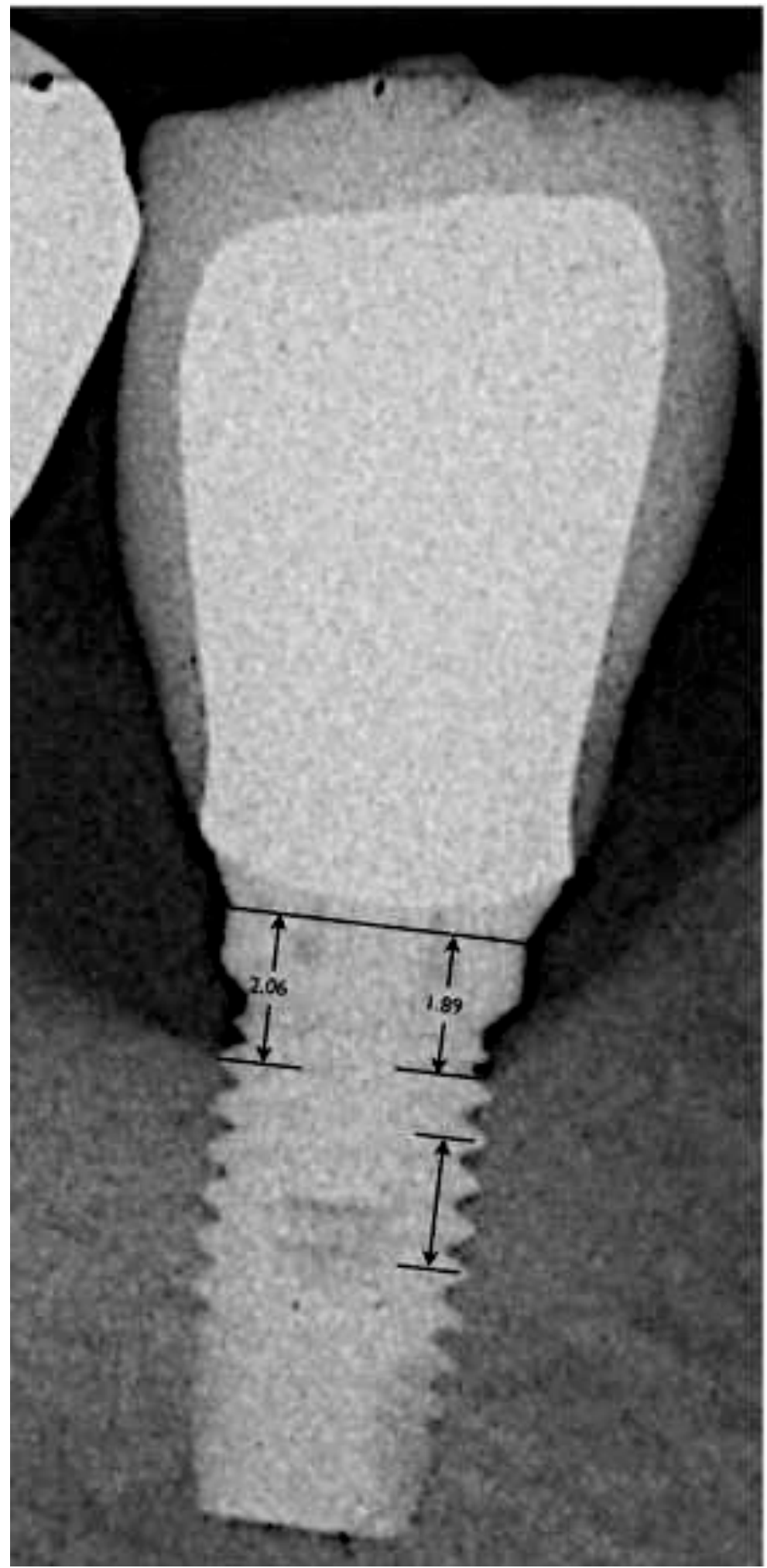

Fig. 1. Measurement of the distance from the top of the implant shoulder to the first visible bone-to-implant contact on digitized radiographs. The distance of three threads was used as a reference for the calibration. 


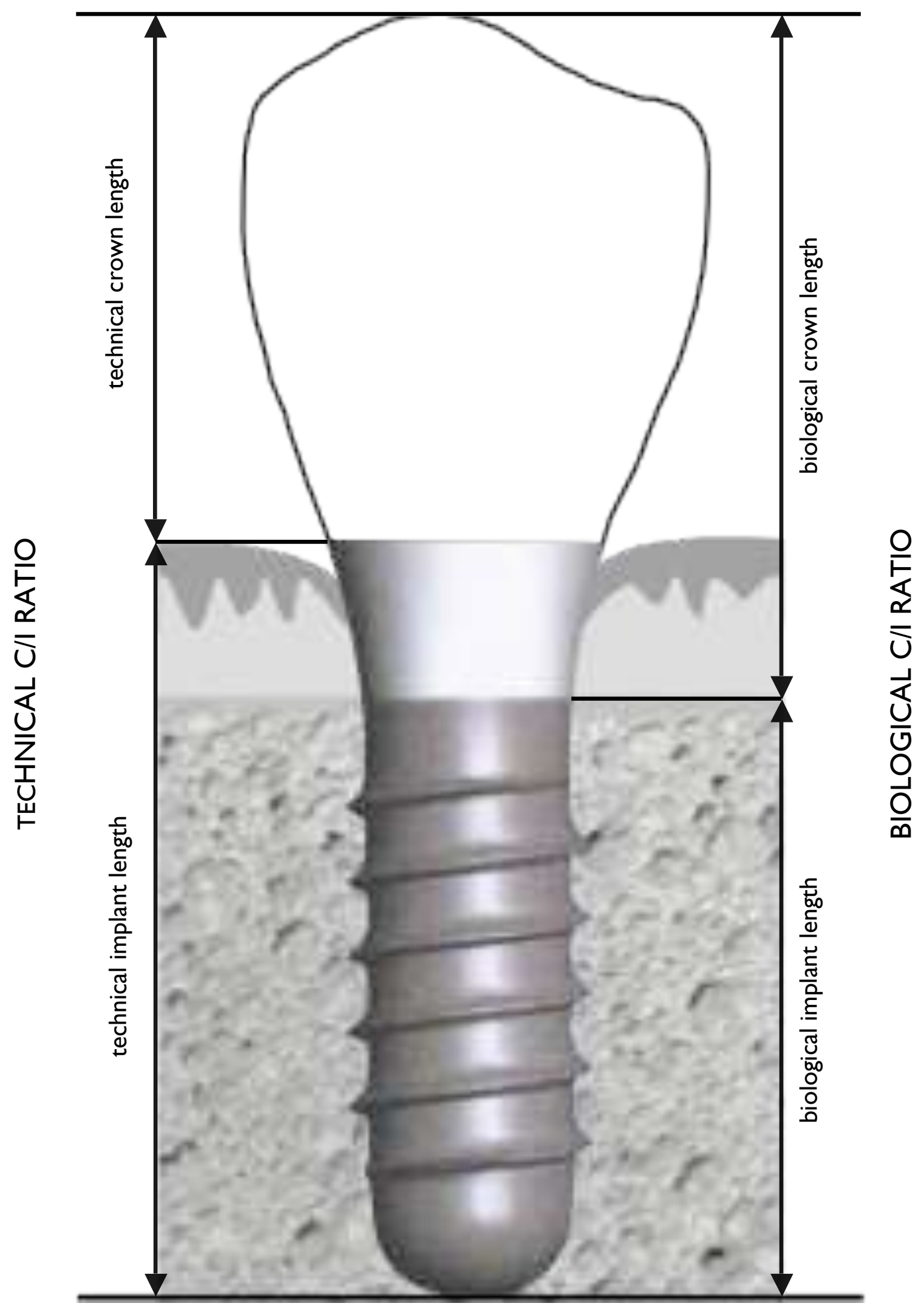

Fig. 2. Assessment of the technical and biological c/i ratio (adapted from Blanes, Bernard et al. 2007). 


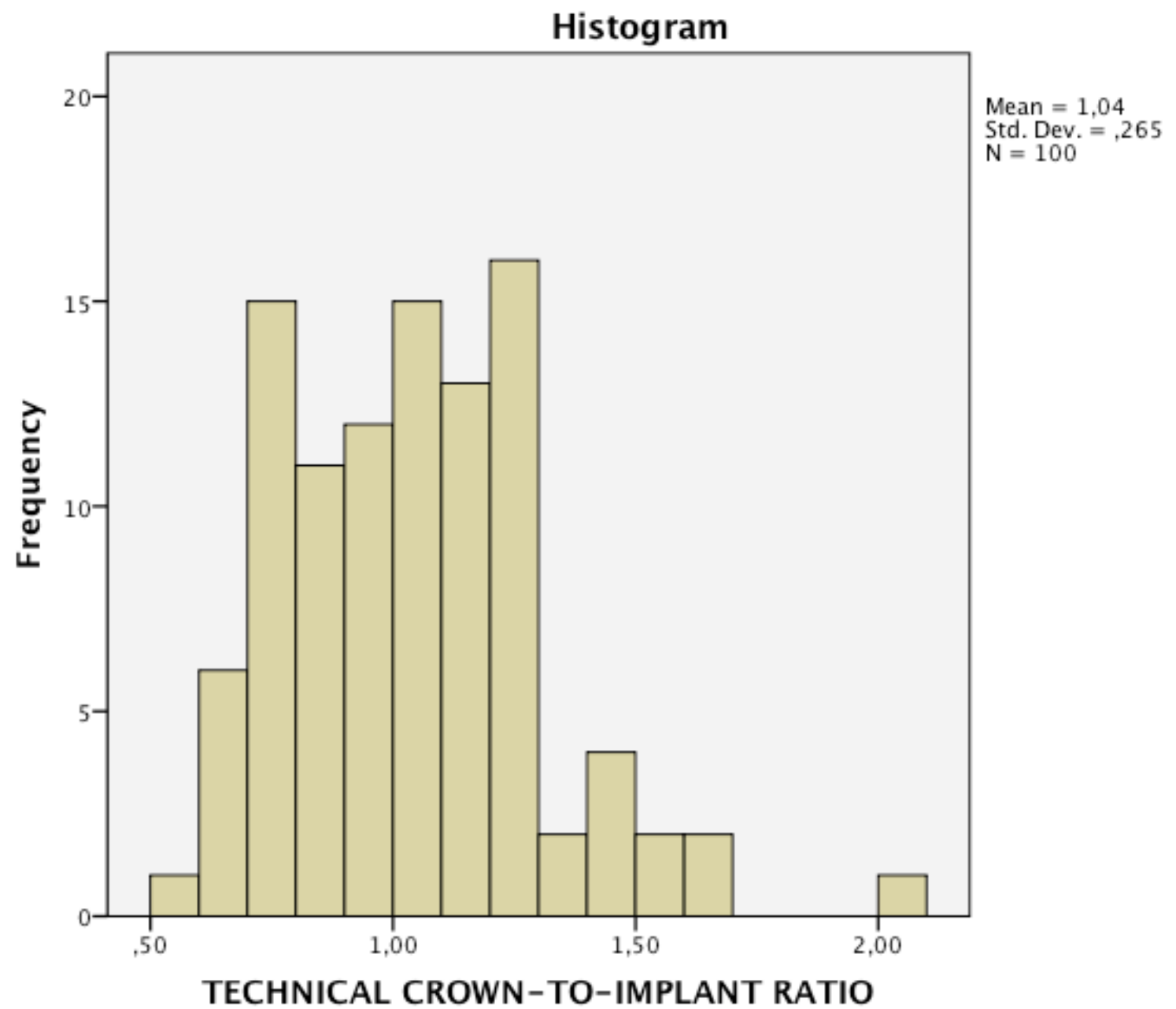

Fig. 3a. Distribution of the implants according to their technical c/i ratio 


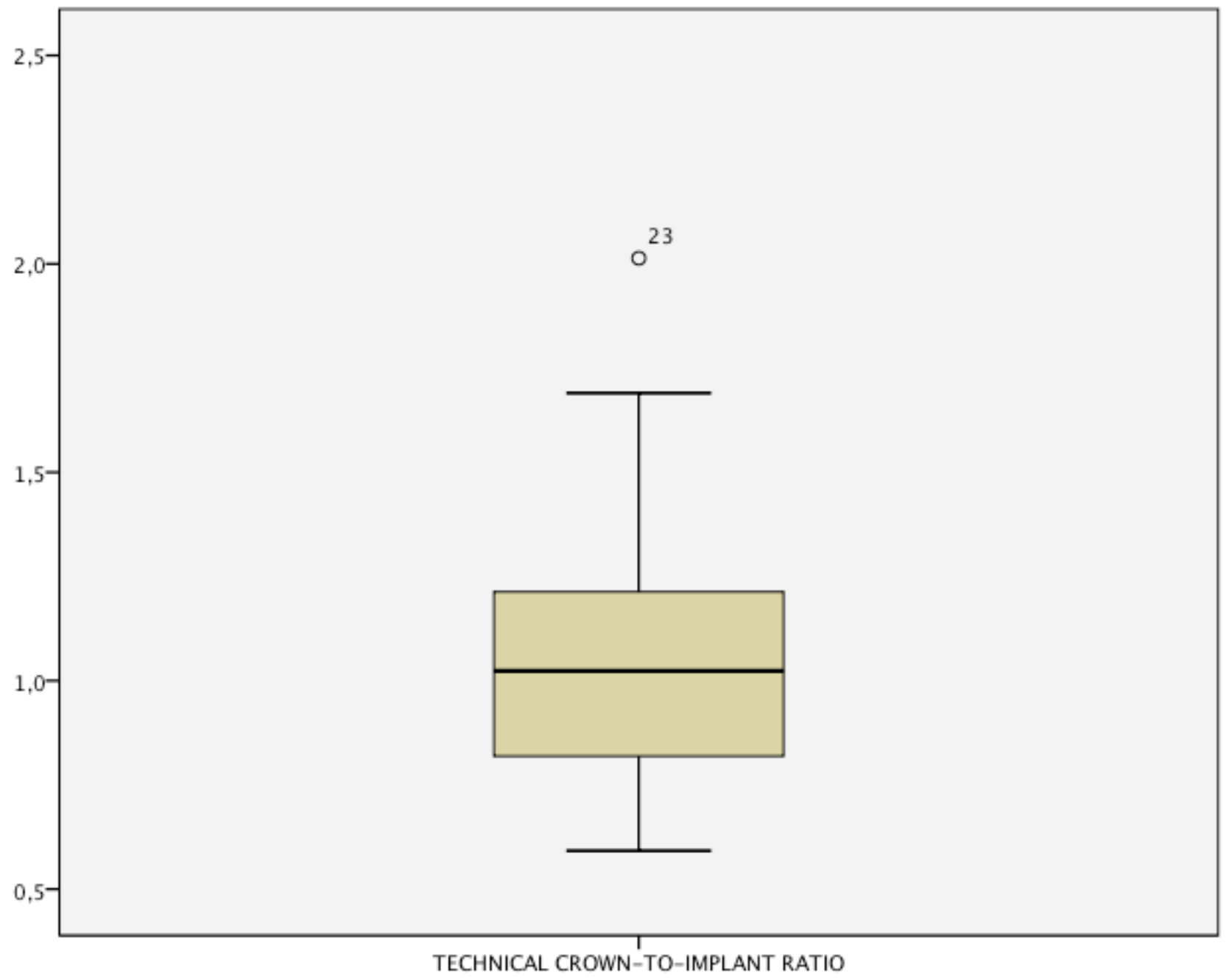

Fig. 3b. Distribution of the implants according to their technical c/i ratio 


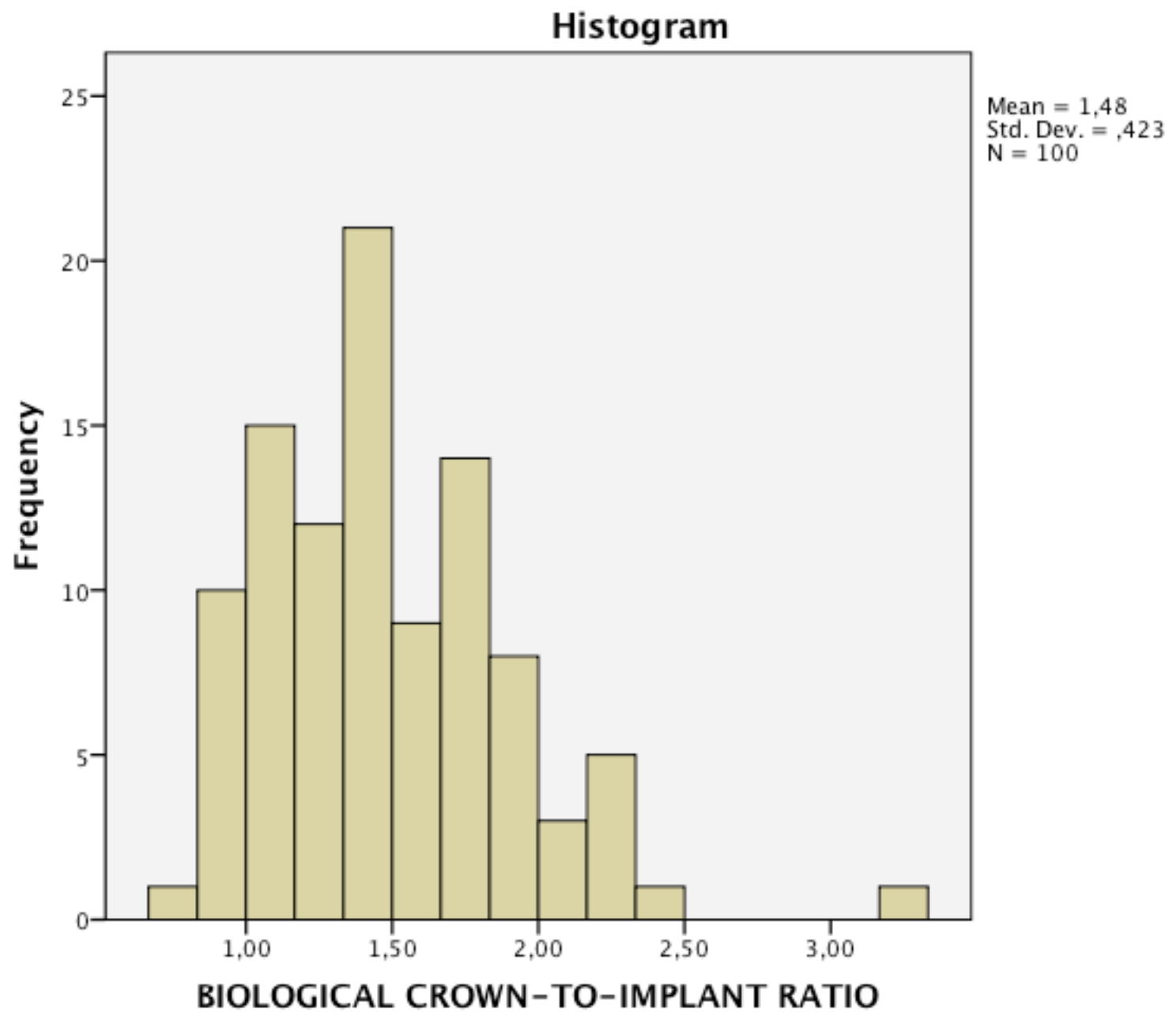

Fig. 4a. Distribution of the implants according to their biological c/i ratio 


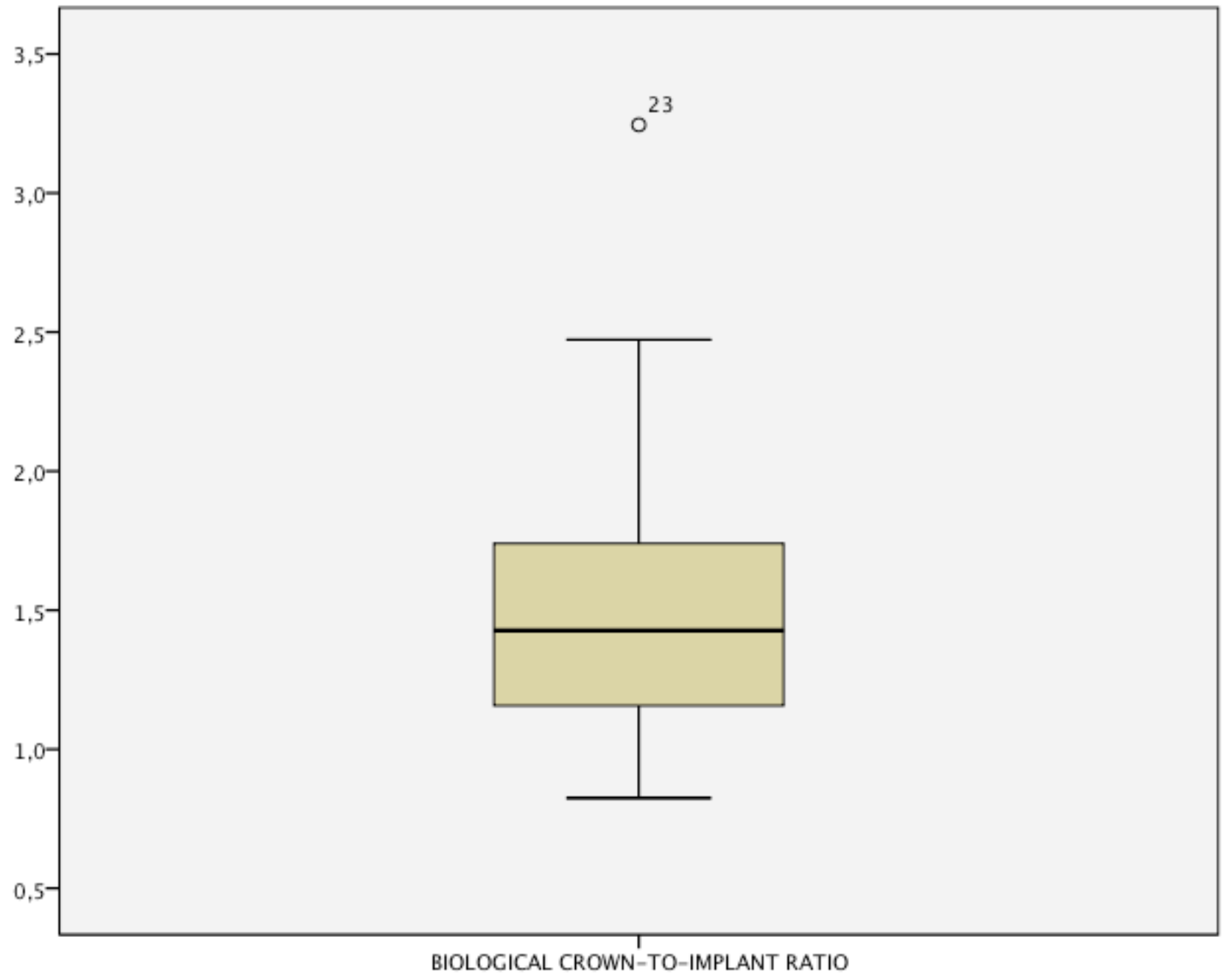

Fig. 4b. Distribution of the implants according to their biological c/i ratio 


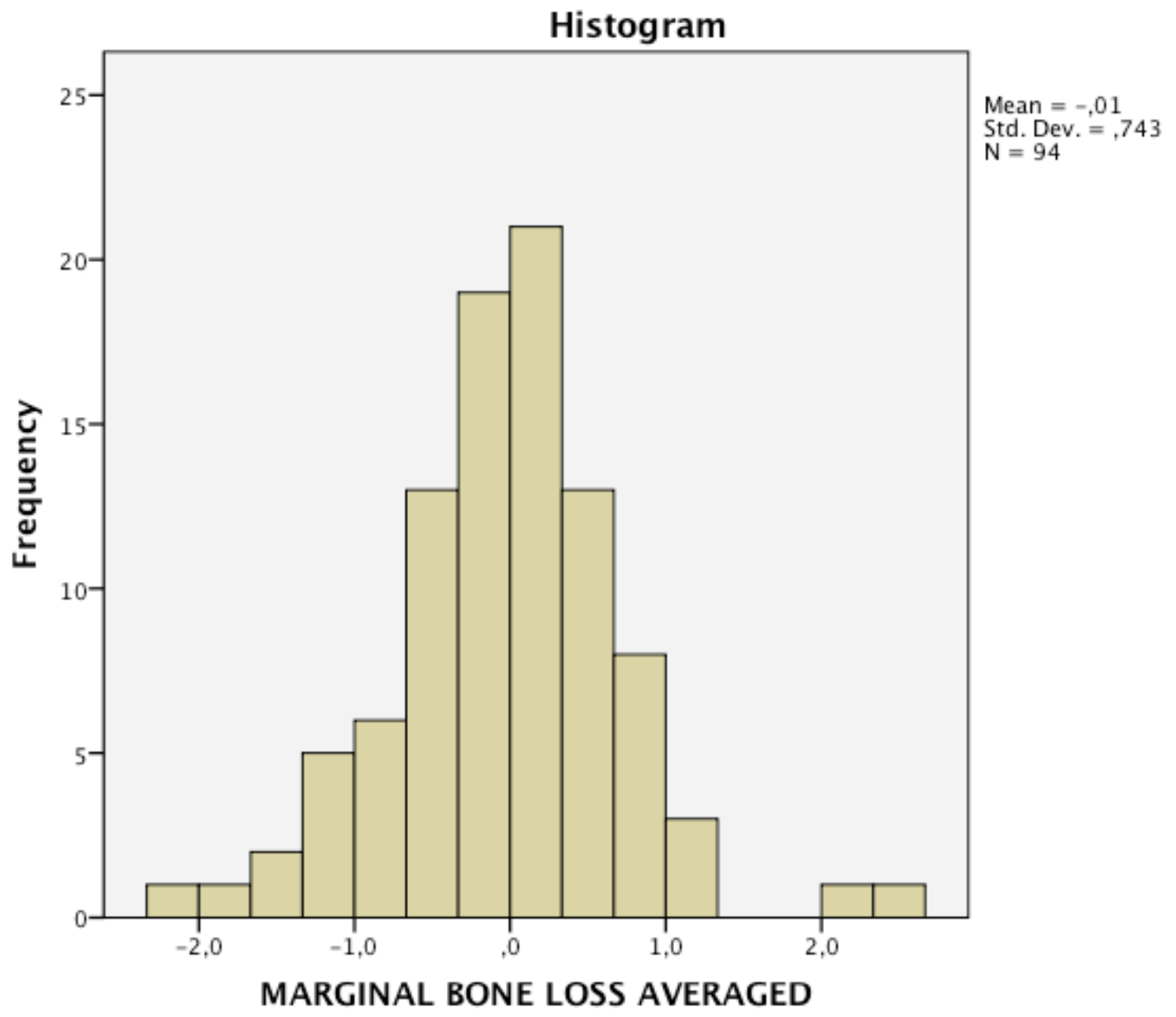


Fig. 5. Distribution of the implants according to their averaged marginal bone loss (millimeters) after 5 years in function

\begin{tabular}{lcc}
\hline type of technical complication & $\mathbf{n}(\%)$ of patients & $\mathbf{n}(\%)$ of implants \\
\hline loss of retention & $5(7.1 \%)$ & $5(5 \%)$ \\
occlusal screw loosening & $4(5.7 \%)$ & $4(4 \%)$ \\
abutment screw loosening & $4(5.7 \%)$ & $4(4 \%)$ \\
chipping of veneering material & $4(5.7 \%)$ & $4(4 \%)$ \\
\hline
\end{tabular}

Table 1. Distribution of technical complications 


\section{References}

Ante, I. (1926). The fundamental principles of abutments. Michigan State Dental Society Bulletin 8: 14-23.

Araujo, M.G. \& Lindhe, J. (2005). Dimensional ridge alterations following tooth extraction. An experimental study in the dog. Journal of Clinical Periodontology 32: 212-218.

Benic, G. I., Jung, R.E. (2009). Clinical and radiographic comparison of implants in regenerated or native bone: 5-year results. Clinical Oral Implants Research 20: 507513.

Blanes, R. J. (2009). To what extent does the crown-implant ratio affect the survival and complications of implant-supported reconstructions? A systematic review. Clinical Oral Implants Research 20 (Suppl. 4): 67-72.

Blanes, R. J., Bernard, J. P. (2007). A 10-year prospective study of ITI dental implants placed in the posterior region. II: Influence of the crown-to-implant ratio and different prosthetic treatment modalities on crestal bone loss. Clinical Oral Implants Research 18: 707-714.

Bornstein, M. M., Schmid, B. (2005). Early loading of non-submerged titanium implants with a sandblasted and acid-etched surface. 5-year results of a prospective study in partially edentulous patients. Clinical Oral Implants Research 16: 631-638.

Buser, D., Weber, H. P. (1991). Tissue integration of one-stage ITI implants: 3-year results of a longitudinal study with Hollow-Cylinder and Hollow-Screw implants. International Journal of Oral \& Maxillofacial Implants 6: 405-412.

Georgiopoulos, B., Kalioras, K. (2007). The effects of implant length and diameter prior to and after osseointegration: a 2-D finite element analysis. Journal of Oral Implantology 33: 243-256.

Gruica, B., Wang, H. Y. (2004). Impact of IL-1 genotype and smoking status on the 
prognosis of osseointegrated implants. Clinical Oral Implants Research 15: 393400.

Jung, R. E., Pjetursson, B. E. (2008). A systematic review of the 5-year survival and complication rates of implant-supported single crowns. Clinical Oral Implants Research 19: 119-130.

Koca, O. L., Eskitascioglu, G. (2005). Three-dimensional finite-element analysis of functional stresses in different bone locations produced by implants placed in the maxillary posterior region of the sinus floor. Journal of Prosthetic Dentistry 93: 3844.

Laurell, L., Lundgren, D. (1991). Long-term prognosis of extensive polyunit cantilevered fixed partial dentures. Journal of Prosthetic Dentistry 66: 545-552.

Lulic, M., Bragger, U. (2007). Ante's (1926) law revisited: a systematic review on survival rates and complications of fixed dental prostheses (FDPs) on severely reduced periodontal tissue support. Clinical Oral Implants Research 18 (Suppl 3): 63-72.

Nyman, S. \& Ericsson, I. (1982). The capacity of reduced periodontal tissues to support fixed bridgework. Journal of Clinical Periodontology 9: 409-414.

Ortorp, A. \& Jemt, T. (2004). Clinical experiences of computer numeric control-milled titanium frameworks supported by implants in the edentulous jaw: a 5-year prospective study. Clinical Implant Dentistry and Related Research 6: 199-209.

Pierrisnard, L., Renouard, F. (2003). Influence of implant length and bicortical anchorage on implant stress distribution. Clinical Implant Dentistry and Related Research 5: 254-262.

Rangert, B. R., Sullivan, R. M. (1997). Load factor control for implants in the posterior partially edentulous segment. International Journal of Oral \& Maxillofacial Implants 12: $360-370$.

Rodoni, L. R., Glauser, R. (2005). Implants in the posterior maxilla: a comparative clinical 
and radiologic study. International Journal of Oral \& Maxillofacial Implants 20: 231237.

Rokni, S., Todescan, R. (2005). An assessment of crown-to-root ratios with short sintered porous-surfaced implants supporting prostheses in partially edentulous patients. International Journal of Oral \& Maxillofacial Implants 20: 69-76.

Schropp, L., Wenzel, A. (2003). Bone healing and soft tissue contour changes following single-tooth extraction: a clinical and radiographic 12-month prospective study. International Journal of Periodontics \& Restorative Dentistry 23: 313-323.

Schulte, J., Flores, A. M. (2007). Crown-to-implant ratios of single tooth implant-supported restorations. Journal of Prosthetic Dentistry 98: 1-5.

Strietzel, F. P., Reichart, P. A. (2007). Smoking interferes with the prognosis of dental implant treatment: a systematic review and meta-analysis. Journal of Clinical Periodontology 34: 523-544.

Tawil, G., Aboujaoude, N. (2006). Influence of prosthetic parameters on the survival and complication rates of short implants. International Journal of Oral \& Maxillofacial Implants 21: 275-282.

Updegrave, W. J. (1968). Right-angle dental radiography. Dental Clinics of North America Nov: $571-579$.

Weber, H. P., Buser, D. (1992). Radiographic evaluation of crestal bone levels adjacent to nonsubmerged titanium implants. Clinical Oral Implants Research 3: 181-188.

Wennstrom, J. L., Ekestubbe, A. (2005). Implant-supported single-tooth restorations: a 5year prospective study. Journal of Clinical Periodontology 32: 567-574.

Yi, S. W., Ericsson, I. (1995). Long-term follow-up of cross-arch fixed partial dentures in patients with advanced periodontal destruction. Evaluation of the supporting tissues. Acta Odontologica Scandinavica 53: 242-248. 